Ulrike Donat

\title{
Freiheitsentziehung im polizeilichen Selbstvollzug am Beispiel der Castor-Transporte
}

\section{Sachverbalt}

Anläßlich des Glaskokillentransportes in 6-Castor-Behältern in das Zwischenlager Gorleben im März 1997 erließ die Bezirksregierung Lüneburg ein mehrtägiges pauschales Versammlungsverbot entlang der Transportstrecke, so Meter rechts und links der Transportstrecke sowie soo Meter um den Verladekran und um das Zwischenlager in Gorleben. Das per Allgemeinverfügung ergangene Versammlungsverbot wurde sowohl von der zuständigen Versammlungsbehörde, dem Landkreis Lüchow-Dannenberg, als auch von der Bürgerinitiative und anderen Betroffenen angefochten. Im Eilverfahren wurde das Verbot vom niedersächsischen Oberverwaltungericht bestätigt, das Hauptsacheverfahren ist in I. Instanz anhängig (VG Lüneburg 7 A 40/97). Tausende von Atomkraftgegnern hatten sich entlang der Transportstrecke in verschiedenen Camps versammelt. Die Camps lagen mit einer Ausnahme außerhalb des räumlichen Geltungsbereiches der Allgemeinverfügung.

Nachdem am Sonntag, den 2.3.1997, im Anschluß an eine nicht verbotene Trekkerdemonstration von soo Treckern und Tausenden Demonstrationsteilnehmern ca. so landwirtschaftliche Maschinen eine Treckerblockade in Splietau errichtet hatten die während der gesamten Aktionstage von der Polizei geduldet wurde! -, kam es ab Montag, den 3.3. 1997, zu vielen Versuchen kleinerer Gruppen, die andere mögliche Transportstrecke zu erreichen. Verschiedentlich setzten sich kleine Gruppen von Schülern oder anderen Personen friedlich auf die Straße, manchmal wurden auch einige Äste auf die Straße geräumt. Daraufhin zog die Polizei am Montag, den 3. 3. 1997, in einem Großaufgebot auf die noch offene Nordstrecke und sicherte diese durch tagelange weiträumige Absperrungen - selbst für Landkreisbewohner - sowie massivste Polizeipräsenz. Hunderte von Polizeifahrzeugen säumten die Strecke. Im Vorfeld war über den Polizeifunk erklärt worden, die "Hamburger Hafenstraße» sammele sich im Quickborner Camp, Autonome würden zum Krieg rüsten. Es handelte sich um nicht belegte Informationen, die auch durch den weiteren Verlauf der Ereignisse keine Nahrung bekamen.

Die Polizei reagierte jedoch durchgehend besonders nervös auf die Versuche aus den Camps in Splietau und Quickborn, Protestaktionen an der Strecke durchzuführen. Am 4. 3. $1997 \mathrm{kam}$ in den Vormittagsstunden die Nachricht über Funk, einige Beamte seien verletzt worden. Tatsächlich hat nur ein Polizeibeamter eine Verletzung angezeigt, die jedoch nicht besonders erheblich war. Angeblich sei ein Stein geflogen.

Daraufhin wurden in einem massiven Polizeieinsatz 569 Personen im Wald bei Quickborn/Kacherin zusammengetrieben und eingekesselt. Dies geschah weit außerhalb der Versammlungsverbotszone, ca. 250 Meter entfernt von der Straße. Auch kleinere Gruppen, die erkennbar am Rand standen, gerade erst ankamen, musizierten oder erkennbar harmlos waren, wurden mit in den Kessel einbezogen. Die Einkesselung erfolgte ca. gegen i 2.30 Uhr. Alleiniger Vorwurf gegen alle s69: Ein Beamter sei verletzt.

In der Folgezeit wurden ca. I go Personen, zum Teil sehr rüde, aus dem Kessel abgeführt, die Personalien festgestellt, Videoaufnahmen und Polaroidfotos gefertigt, Kabelbinder (Plastikfesseln) angelegt. Diese i so Personen wurden in die Gefange- 
nensammelstelle nach Neu Tramm verbracht. Ein Pastor begleitete den Transport, um dafür zu sorgen, daß die sehr fest angezogenen Plastikfesseln frühzeitig entfernt würden.

Vor Ort in Quickborn bemühten sich Landtagsabgeordnete, Pastoren und Rechtsanwälte um Vermittlung und Deeskalation. Nach Stunden wurde erreicht, daß im Wald lediglich eine Personalienfeststellung erfolgen und danach die Betroffenen freigelassen werden sollten. Die Personalienfeststellung erfolgte trotz Protesten mit Videokamera. Am späten Nachmittag kamen die letzten frei (ca. zwischen I 6.00 und $17.00 \mathrm{Uhr}$ ). Alle bekamen anschließend ein Ermittlungsverfahren wegen schweren Landfriedensbruchs ( $\$$ I 25 a $\mathrm{StBG}$ ), sämtlichst gestützt auf den Pauschalvorwurf, ein Polizeibeamter sei verletzt worden. Bereits in der Anzeige wurden keinerlei Zurechnungskriterien angegeben. Dementsprechend wurden sämtliche Ermittlungsverfahren später eingestellt. Sie hatten offensichtlich den Sinn, die "Straftatenbilanz « für die Medien zu erzeugen.

Anders erging es den ca. I 50 Betroffenen, die nach Neu Tramm verbracht wurden: Vor Ort befindliche Anwälte bemühten sich sofort um die Gefangenen; dennoch kam es wegen erheblicher organisatorischer Schwierigkeiten in der Gefangenensammelstelle zu mehrstündigen Verzögerungen. Obwohl bereits bei der Zuführung zum Gefangenenbus die Identitätsfeststellung erfolgt war, wurde erneut von der Kriminalpolizei eine erkennungsdienstliche Behandlung durchgeführt (Namen, Foto, zum Teil Videoaufnahmen). Sie wurden bei der Kriminalpolizei »wegen Verdacht einer Straftat zum Nachteil des Polizisten xy « vernommen.

Nach dieser Vernehmung wurden die Betroffenen einzeln wieder in die Gefangenensammelstelle geführt und dort zusammen mit den noch nicht vernommenen zusammengesperrt. Erst nachdem feststand, daß strafrechtliche Vorwürfe nicht konkretisiert werden konnten, wurde dann dort der Polizeigewahrsam »zur Verhinderung von Straftaten" nach dem Niedersächsischen Gefahrenabwehrgesetz (NGefAG) angeordnet mit der gleichen Begründung wie zuvor, die Menge habe unter Zuhilfenahme gewalttätiger Mittel einen Beamten angegriffen und versucht, die Versammlungsverbotszone zu erreichen.

Von Anfang an war der zuständige Richter - der gleichzeitig als Haftrichter fungierte - vor Ort. Dennoch wurden alle Betroffenen rund I 2 Stunden ohne jede richterliche Entscheidung festgehalten. Mehrere Anwälte intervenierten, der Richter hatte jedoch von der Polizei keinerlei Akten erhalten und fühlte sich außerstande, eine Entscheidung zu treffen. Nach stundenlangen Verhandlungen wurde lediglich erreicht, daß alle unter I8- oder über 60jährigen vorzeitig entlassen wurden. Erst ca. Io Stunden nach Beginn der Einkesselung, also gegen ca. 21.30 Uhr, wurde dem zuständigen Richter die erste Akte vorgelegt. Diese Akte enthielt lediglich den erwähnten Pauschalvorwurf, ein Beamter sei verletzt, ohne jeden individualisierten Tatbeitrag. Dasselbe Vorblatt wurde fotokopiert für I 50 Vorgänge verwandt! Der Richter verfügte gegen 24.00 Uhr die Freilassung, die sich dann aber noch bis I.45 Uhr hinstreckte.

Bis heute konnten für keinen einzigen der Betroffenen konkretisierte Straftatenvorwürfe genannt werden außer einem Fall von verbotener Vermummung (dieses Verfahren wurde eingestellt gegen Geldbuße). $\mathrm{Zu}$ vermuten ist jedoch, daß alle 569 Betroffenen unterschiedslos in einer sogenannten "Störerdatei« erfaßt sind. Vor Ort wurde gegen den Leiter der kriminalpolizeilichen Abteilung in der Gefangenensammelstelle Neu Tramm Strafanzeige wegen Freiheitsentziehung gestellt, weil dieser den »Anschlußgewahrsam« angeordnet hatte, nachdem feststand, daß eine Freiheitsentziehung nach StPO nicht gerechtfertigt war.

Vor Ort wurde von den Betroffenen über ihre Anwälte ebenfalls Antrag auf gericht- 
liche Entscheidung gestellt. Das Amtsgericht Dannenberg hat durch Beschluß vom 21.3. I997, Az. 10 XIV 100/97 entschieden und die rund I2-stündige Ingewahrsamnahme damit begründet, daß hätte geprüft werden müssen, ob eine Ingewahrsamnahme stattfinden müsse.

Das Landgericht in Lüneburg hat durch Beschluß vom 10. I 2. 1997, Az. I D 77/97, die Anträge zurückgewiesen, weil nach seiner Auffassung kein Antrag auf nachträgliche Überprüfung der Freiheitsentziehung, sondern nur ein Antrag auf gerichtliche Entscheidung während der Freiheitsentziehung vorlag. Der genaue Sachverhalt kann jedoch nicht geklärt werden, da aufgrund der chaotischen Zustände in der Gefangenensammelstelle die Anträge zwar zu Protokoll gegeben wurden, jedoch die Protokollierung nicht ordnungsgemäß erfolgte. Das Gericht residiert in Form des Richters bei Castor-Transporten in der Polizeikaserne, und zwar ohne Geschäftsstellenbeamte oder Protokollführer. Das Hausrecht, Verwaltungsvorgänge usw. werden von Polizeikräften ausgeübt. Für Freiheitsentziehungsverfahren wird inzwischen zwar eine eigene Akte angelegt, jedoch wird diese von Polizeikräften und nicht von Gerichtspersonal erstellt.

\section{Rechtliche Würdigung}

Die Freiheitsentziehung von 569 Personen wegen eines Pauschalvorwurfes war insgesamt rechtswidrig. Sie verstößt vor allem gegen die Kriterien, die die höchstrichterliche Rechtsprechung für die Zurechnung von gewalttätigen Ausschreitungen einzelner für andere, friedliche Demonstrationsteilnehmer entwickelt hat (insbesondere: Brokdorf-Beschluß des Bundesverfassungsgerichtes, NJW I985, 2395; Bundesgerichtshof im Grohnde-Urteil, NJW 1984, I226, und zum Schadensersatz bei Demonstrationen, BGH-Urteil vom 4. I I. I997, VI ZR 348/96).

Insgesamt ist der Quickborner Kessel ein Beispiel für den um sich greifenden unrühmlichen »instrumentellen Selbstvollzug « der Polizei. Das Mittel des Polizeigewahrsams wird benutzt, um eine Bestrafung der DemonstrantenInnen herbeizuführen, wenn das Beweismaterial für eine gerichtliche Verurteilung oder gar einen Haftbefehl nicht ausreicht. Zudem wird mittels pauschalisierter Zurechnung umfassendes Datenmaterial über Demonstrationsteilnehmer gesammelt. Die Vernichtung der Videoaufnahmen, der Personalien und Fotografien zu erreichen, ist im Nachhinein weitaus schwieriger, als die Einstellung eines Ermittlungsverfahrens. Bedenklich ist auch der Umgang mit den Versammlungsverboten: In den verwaltungsgerichtlichen Verfahren wurde die 5o-Meter-Zone stets für ausreichend konkretisiert erachtet, obwohl bereits in den Vorjahren vorgetragen wurde, daß dieses Kriterium willkürlich benutzt wird, die BürgerInnen im gesamten Landkreis Lüchow-Dannenberg umfassenden Polizeikontrollen und Polizeieingriffen zu unterwerfen, und daß ein abgrenzbares Kriterium in Wald und Flur fehlt. Auch in Parallelfällen wurde bei Polizeimaßnahmen außerhalb der Verbotszone stets von der Polizei zur Rechtfertigung angeführt, die Betroffenen seien auf dem Weg, um das Versammlungsverbot zu verletzen. Dieser Umgang mit einem angeblich bestimmten Abgrenzungskriterium führt dieses Kriterium ad absurdum.

Usus wird auch die willkürliche Anordnung von »Anschlußgewahrsam « durch die Polizei, wenn und nachdem feststeht, daß eine Freiheitsentziehung nach $₫$ I $28 \mathrm{StPO}$ nicht zulässig ist. Hierbei wird ignoriert, daß auch der polizeiliche Gewahrsam tatbestandlich einen konkreten, personenbezogenen Verdacht einer Straftat oder Ordnungswidrigkeit von erheblichem Gewicht voraussetzt (vgl. \I8 Abs. I NGefAG) und daher nicht vorliegen kann, wenn genau das gleiche Kriterium bereits bei der strafrechtlichen Beurteilung verneint werden mußte. 
Ebenso ungerechtfertigt ist die Verzögerung der Anhörung der Betroffenen bzw. ihrer Vorführung beim Freiheitsentziehungsrichter. Dieser hätte beispielsweise die nach Freiheitsentziehungsrecht vorgeschriebene Anhörung parallel zur kriminalpolizeilichen Bearbeitung durchführen können. Nach obergerichtlicher Rechtsprechung ist nämlich die Dauer der Freiheitsentziehung nach verschiedenen Rechtsgrundlagen nicht kumulativ aneinanderzureihen, sondern es kommt auf die absolute Begrenzung der zulässigen Freiheitsentziehung an (vgl. BGHSt $34,365=\mathrm{NStZ}_{\mathbf{1}} 988$, 333). Sämtliche Rechtsvorschriften verlangen die »unverzügliche« Herbeiführung der richterlichen Entscheidung (vgl. z. B. $\$ \$$ i 9 Abs. I NGefAG, 36 PolGNW, 40 BGSG und Art. ${ }_{0}{ }_{4}$ Abs. 2 GG), von der nach Ablauf von $10^{1 / 2}$ Stunden nicht mehr die Rede sein kann.

Die Polizei ist verpflichtet, entsprechende organisatorische Vorkehrungen zu treffen. Sie darf den Richtervorbehalt nicht durch Hinhalten und Irreführung des Richters außer Kraft setzen.

\section{Rechtliche Fragen der Freibeitsentziebung}

\section{a) Antrag auf gerichtliche Entscheidung wäbrend der Freibeitsentziehung}

Antragsbefugt ist de lege lata nur die Verwaltungsbehörde, also die Polizei, nicht jedoch die Betroffenen selbst (»... hat die Polizei unverzüglich eine richterliche Entscheidung ... herbeizuführen. « - $\$ \$ 36$ I I PolGNW, 18 I I NGefAG, ebenso $\$ 40$ I BGSG).

Ein Antragsrecht der Betroffenen auf gerichtliche Entscheidung ist gesetzlich nicht vorgesehen. So kann die Polizei jedoch den Grundrechtsschutz unterlaufen, wenn sie nicht oder nicht unverzüglich richterliche Entscheidung beantragt. Den Betroffenen bleibt dann nur, auf einer richterlichen Anhörung ( $\$ 5$ FEVG, $70 \mathrm{c}$ FGG) zu bestehen. Hierbei sind sie ggf. der Willkür der Verwaltungsbehörde ausgeliefert, die dann geltend macht, die Akten seien noch nicht fertig, der Computer abgestürzt etc.

Beim Karwitzer Kessel vor dem Castor-Transport nach Gorleben im Mai $1996^{1}$ hat die Polizei erst nach s Stunden den Freiheitsentziehungsrichter benachrichtigt, obwohl sie über I so Personen festgenommen hatte und nur ein Richter verfügbar war. Es wurde nicht ein einziger namentlicher Antrag gestellt und kein einziger Betroffener vorgeführt, weil die Beamten - wahrheitsfremd - behaupteten, es handele sich ausschließlich um eine BGS-Maßnahme.

Beim Quickborner Kessel (s.o.) ${ }^{2}$ dauerte es Stunden, bis die Polizei namentliche Anträge stellte und mit Vorführungen begann. Der Richter sah sich nicht zu einer Entscheidung in der Lage, weil ihm keine Akten vorgelegt wurden.

In Ahaus im Oktober 1997 unterblieb jede Beiziehung eines Richters, dort waren keine Anwälte in der Gefangenensammelstelle. Ca. I30 Personen wurden ca. 9 Stunden ohne richterliche Entscheidung festgehalten ${ }^{3}$.

Im März 1998 im Zusammenhang mit den Ahaus-Protesten wurden Eilrichter von Rechtsanwälten informiert. Obwohl Betroffene bereits über 10 Stunden im Polizeigewahrsam waren und insgesamt ca. 700 Personen festgehalten wurden, hatte die Polizei nicht einen einzigen Antrag auf richterliche Entscheidung gestellt $t^{4}$. Auch hier wurden den Richtern weder Listen mit Namen und Anzahl der Gefangenen und

\footnotetext{
I Vgl. z. B. AG Dannenberg, 3 XIV I61 L/GA (mit 33 Parallelverfahren), LG Lüneburg 4 T 218/96 und I T 30/98 (Verfahren ist noch nicht abgeschlossen) sowie VG Luneburg, $7 \mathrm{~A} \mathrm{60/97} \mathrm{(noch} \mathrm{anhangig).}$

2 Z.B. AG Dannenberg, Io XIV $103 / 97$ (und etliche Parallelverfahren), LG Luneburg I T 74/97 und OLG

Celle 17 W 6/98 sowie VG Luneburg, 7 A 35/98 (noch anhangig).

3 Z.B. VG Münster I K 3632/97 und I K $367 \mathrm{I} / 97$ (noch anhangig).

4 Z.B. VG Munster, I K $1056 / 98$ und I $\mathrm{K} \operatorname{105} 8 / 98$ (noch anhangig).
} 
gegen sie gerichteter Vorwürfe, noch einzelne Akten vorgelegt. Erst auf Druck von

Rechtsanwälten wurde eine Einzelentscheidung gefällt, mit der die Freilassung auch gleichgelagerter Fälle angeordnet wurdes. Dennoch bestand die Polizei darauf, nur nach richterlicher Einzelentscheidung freizulassen, legte aber keine weiteren Akten vor. Einzelanhörungen konnten nur schleppend durchgesetzt werden. Über viele Stunden wurden die Betroffenen daraufhin weiter festgehalten, obwohl das Gericht die Rechtsgrundlage nicht als erfüllt angesehen hatte.

Diese Fälle zeigen, daß de lege ferenda ein Antragsrecht auf richterliche Entscheidung bei Freiheitsentziehungen für die Betroffenen explizit einzuführen ist, damit der Grundrechtsschutz (Art. I04 GG) nicht leerläuft. Auch jetzt schon ist ein Antragsrecht aus der Entscheidung des Bundesverfassungsgerichtes vom 30.4. 1997 (NJW 1997, 2163) abzuleiten, auch soweit es nicht kodifiziert ist (so schon OVG Münster, NJW I990, 3224). Eine Freiheitsentziehung ist immer ein tiefgreifender Grundrechtseingriff, für den effektiver Rechtsschutz nach Art. 19 Abs. 4 GG zu gewähren ist.

\section{b) Anbörung der Betroffenen}

Die mündliche Anhörung der Betroffenen ist gesetzlich vorgeschrieben ( $\$$ s Abs. I FEVG, $\$ 70$ c FGG). Demgegenüber ist es Praxis bei polizeilichen Massenfestnahmen, den Betroffenen nur einen Anhörungsbogen auszuhändigen. Dies genügt dem Erfordernis der mündlichen bzw. persönlichen Anhörung nicht und ist zudem deshalb zweifelhaft, weil nach den bisherigen Erfahrungen in Gorleben und Ahaus die Aktenführung der Polizeibehörden problematisch ist. Teilweise wurden Ermittlungsverfahren gegen Betroffene, das Freiheitsentziehungsverfahren nach Polizeirecht und verwaltungsrechtliche Widersprüche in einer Akte geführt. Da es den Betroffenen jedoch freisteht, sich im Ermittlungsverfahren zu äußern, werden sie in der Regel den Anhörungsbogen nicht ausfüllen. Tun sie es doch, ist nicht sichergestellt, daß dies nur im Freiheitsentziehungsverfahren, nicht aber in anderen Verfahren verwertet wird. Schließlich sehen die Anhörungsbögen nur vor, daß sich die Betroffenen zum Vorwurf (»kam Platzverweis nicht nach« etc.) äußern, nicht jedoch weitere Umstände, die für die Gefahrenprognose und damit die Fortdauer der Freiheitsentziehung erheblich sind, wie z. B. die Absicht, nach Hause zu fahren.

Zudem soll die Anhörung dem Richter einen »unmittelbaren Eindruck « verschaffen und dazu dienen, den Betroffenen über den Gang des Verfahrens und die Grundlagen der Freiheitsentziehung zu informieren ( $\$ 70$ c FGG). Das rechtliche Gehör bei einer schriftlichen Anhörung ist aus den o.g. Gründen nicht gewahrt.

\section{c) Rechtsweg für nachträgliche Überprüfung der Freibeitsentziebung}

Nach der Entscheidung des BayObLG vom 6. 7. 1989 (NVwZ 1990, 1994) beschränkt sich die nachträgliche Überprüfung der Gerichte der freiwilligen Gerichtsbarkeit hinsichtlich einer vor gerichtlicher Entscheidung beendeten Freiheitsentziehung allein darauf, ob die Festhaltung aufgrund Polizeirecht gerechtfertigt war, dagegen kann nicht entschieden werden, ob sie als Strafverfolgungsmaßnahme rechtmäßig war. In diesem Verfahren kann auch nur die Rechtmäßigkeit der Freiheitsentziehung, nicht jedoch die Rechtmäßigkeit der sonstigen polizeilichen Maßnahmen überprüft werden, weshalb die Fortsetzungsfeststellungsklage nach VwGO als Rechtsbehelf vorzuziehen ist.

Strittig ist jedoch, inwieweit der Gang zum Verwaltungsgericht durch die Regelung in

5 Amtsgericht Coesfeld, B. V. 20. 3.1998, 9 XIV 24/98. 
$\$ 19$ Abs. 3 und 4 NGefAG ausgeschlossen ist (offengelassen vom Landgericht Lüneburg, B. v. 25. 10.1996, Az. I T 120/96).

Für die nachträgliche Überprüfung der Rechtmäßigkeit der Ingewahrsamnahme hat das Verwaltungsgericht Lüneburg (B. v. 6. 5. 1997, Az. 7 A 50/97) entschieden, das Amtsgericht sei auch hierfür ausschließlich zuständig, wenn bereits eine richterliche Entscheidung über die Freiheitsentziehung erfolgt sei, selbst wenn der Beschwerdeweg ausgeschöpft sei. Deshalb sei auch eine Fortsetzungsfeststellungsklage nach $\mathbb{S}$ I 3 Abs. I S. 4 VwGO nicht zulässig (vgl. auch VGH Kassel, NJW I984, 82 Iff.). $\mathbb{\$} 19$ As. 2 NGefAG enthalte jedoch nur eine engbeschränkte Ausnahmeregelung, während in allen nicht geregelten Fällen weiter die VwGO gelte (B. V. 5. 5. 1996, I T I20/96). Demgegenüber hat das Niedersächsische Oberverwaltungsgericht die $\mathrm{Zu}$ ständigkeit des Verwaltungsgerichtes bejaht, wenn nicht die "Zulässigkeit und Fortdauer « der Freiheitsentziehung und nicht die Frage der Rechtmäßigkeit einer vor richterlicher Entscheidung beendeten Freiheitsentziehung im Streit ist, sondern der Antrag auf Ingewahrsamnahme durch die Verwaltungsbehörde (hier die Polizei) und die Rechtmäßigkeit des amtsgerichtlichen Beschlusses zur Ingewahrsamnahme nachträglich Streitgegenstand ist (B v. 2.7. 1997, Az. ${ }_{3} \mathrm{O}$ 2664/97).

Soweit eine gesetzliche Rechtswegzuweisung im Polizeigesetz nicht besteht, ist die Rechtslage ebenfalls uneindeutig: Das OLG Köln hat (zur Abschiebehaft) entschieden, daß auch nach Beendigung freiheitsentziehender Maßnahmen das Rechtsschutzinteresse fortbestehe und daher auch nachträglich sofortige Beschwerde und weitere sofortige Beschwerde im FGG-Verfahren zulässig seien (B. V. 16.7. 1997, NJW 1998, 462 unter Hinweis auf BVerfG B.v. 19.6.1997, EuGRZ 1997, 372 und vom 26.6.1997, EuGRZ 1997, 374). Dagegen hält das OLG Hamm nach Beendigung der Beschwer Rechtsmittel der freiwilligen Gerichtsbarkeit für unzulässig und schließt jedenfalls eine nachträgliche Überprüfung der Rechtmäßigkeit der Maßnahme im FGG-Verfahren aus (B. v. 24.1 1. 1997, NJW 1998, 463).

Nach OVG Münster sind für die nachträgliche Rechtmäßigkeitskontrolle die ordentlichen Gerichte weiterhin zuständig, wenn sie während der Freiheitsentziehung bereits angerufen wurden (NJW 1990, 3224), auch wenn die richterliche Maßnahme bestimmten Mindestanforderungen nicht genügte (a. A. VGH Kassel, NJW I984, 821 ). Ist hingegen der Freiheitsentziehungsrichter nicht angerufen worden, obliegt die richterliche Kontrolle nach Entlassung aus dem Gewahrsam den Verwaltungsgerichten. Diese Einschränkung des Verwaltungsrechtsweges überzeugt nicht. Vielmehr ist hier mit der oben zitierten Entscheidung des Niedersächsischen Oberverwaltungsgerichtes (B. v. 2.7. 1997, I3 O 2664/97) in allen Fällen die Zulässigkeit der Fortsetzungsfeststellungsklage nach $₫ \mathrm{I}_{3} \mathrm{Abs}$. I S. 4 VwGO anzunehmen, denn der Richtervorbehalt ist nach allen Erfahrungen in Eilsachen nicht geeignet, den Grundrechtsschutz zu sichern (vgl. hierzu Asbrock, Der Richtervorbehalt - prozedurale Grundrechtssicherung oder rechtsstaatliches Trostpflaster?, ZRP 1998, i7 ff.).

\section{d) Durchsetzung des Grundrechtsschutzes}

Handlungsmöglichkeiten für Betroffene und Anwälte sind

aa) während der Freiheitsentziehung:

- Antrag auf richterliche Entscheidung, ggf. selbst den zuständigen Richter benachrichtigen

- mündliche Anhörung herbeiführen, ggf. auch ohne Aktenvorlage der Polizei, denn neben den Inhaftierungsgründen kommt es auch auf die Gefahrenprognose für die Fortdauer der Freiheitsentziehung an

- die Einsatzleitung kann auf $\$ 239$ StGB hingewiesen werden. 
bb) nach Entlassung aus dem Gewahrsam:

- Fortsetzungsfeststellungsklage nach $\$$ II $3_{3} \mathrm{VwGO}$ oder

- Antrag auf nachträgliche Überprüfung der Freiheitsentziehung nach $\ 19$ Abs. 2 NGefAG oder

- analog BVerfG, NJW 19972163 Antrag auf nachträgliche Überprüfung der Freiheitsentziehung im FGG- bzw. StPO-Verfahren (str.)

Hierbei sind die Vorteile des verwaltungsgerichtlichen Verfahrens (Kollegialgericht, unfassendere Sachverhaltserforschung, solide Kenntnisse des Polizeirechtes, Öffentlichkeit, Überprüfung aller polizeilichen Maßnahmen) abzuwägen gegen die Risiken der oben geschilderten Unklarheiten zur Rechtswegzuweisung.

De lega lata sind zu fordern die eindeutige Aufnahme des Antragsrechtes der Betroffenen auch während des Gewahrsams sowie klare Rechtswegzuweisung für alle nachträglichen gerichtlichen Kontrollen an die Verwaltungsgerichtsbarkeit als sachnächstem Instanzenzug.

\section{Martin Kutscha »Schlanker Staat« mit Januskopf}

\section{- Ein Zwischenruf -}

»Weniger Staat - mehr bürgerliche Freiheit«, lautet die Quintessenz im Abschlußbericht des 1995 von der Bundesregierung eingesetzten Sachverständigenrates »Schlanker Staat $\aleph^{1}$. Die Forderung klingt sympathisch. Nahezu jeder, quer durch alle parteipolitischen Fronten, bekennt sich inzwischen zur Option einer Verschlankung des Staates, wie der Vorsitzende des Sachverständigenrates, Rupert Scholz, denn auch mit Genugtuung feststellen konnte 2 . In der Tat: Die meisten der auch von anderer Seite vorgeschlagenen und seit Jahren zielstrebig umgesetzten Schritte zur Verwirklichung des "Schlanken Staates « werden von breiter gesellschaftlicher Akzeptanz getragen. Erinnert sei nur an die (auch durch entsprechende Werbung angekurbelte) Euphorie von Kleinanlegern beim Gang der privatisierten Deutschen Telekom AG an die Börse. Dabei sind Post und Bahn zwar die bedeutsamsten, aber nicht die einzigen Bereiche ehemals staatlicher Infrastrukturleistung, die inzwischen in private Hände überführt wurden ${ }^{3}$. Während es allerdings bei manchen Privatisierungsmaßnahmen schlicht um die kurzfristige Deckung von Haushaltsdefiziten geht (so z. B. beim Verkauf von Eigenbetrieben des Landes Berlin), steht für den Sachverständigenrat die »ordnungspolitisch gebotene Weichenstellung « im Vordergrund: "Privates Eigentum und privatwirtschaftliche, durch Markt und Wettbewerb gesteuerte wie kontrollierte unternehmerische Tätigkeit gewährleistet am besten wirtschaftliche Freiheit, ökonomische Effizienz und Anpassung an sich verändernde

I Sachverstandigenrat »Schlanker Staat«, Abschlußbericht, Bd. I, Bonn o. J. (1997), S. Is.

2 A.a.O., S. 5.

3 Zu den verschiedenen Privatisierungsformen sowie zur Problematik im einzelnen vgl. T. Blanke/D. Sterzel, Ab die Post? KJ 1993, 278; L. Osterloh, Privatisierung von Verwaltungsaufgaben, VVDStRL 54 (1994), 204; die Beitràge von G. F. Schuppert u. a. in: K. König/A. Benz (Hrsg.), Privatisierung und staatliche Regulierung, Baden-Baden 1997; M. Kutscha, Verfassungsfragen der Privatisierung von Staatsaufgaben, NJ r 997, 393 